\title{
Effects of Helicity and System Rotation on Decaying Homogeneous Turbulence*
}

\author{
Youhei MORINISHI**, Koichi NAKABAYASHI** \\ and Shuiqiang REN**
}

\begin{abstract}
To consider the nonreflection property of the turbulence statistics in a rotating system, a new method has been proposed to introduce turbulence helicity into an isotropic turbulent field for direct numerical simulation (DNS). Effects of the helicity and the rotation on turbulence statistics and vortex structure are investigated by theoretical analysis and DNS of the homogeneous decaying turbulence. Although DNS results show that both the rotation and the helicity inhibit energy decay, theoretical analysis reveals the occurrence of very different mechanisms for the rotation and the helicity for producing the inhibition effect. The helicity directly decreases the energy transfer while the rotation suppresses the energy transfer due to the so-called scrambling effect. The other effect of system rotation is that rotation elongates the vortex structure along the rotation axis, however, the presence of the helicity appears to weaken this tendency.
\end{abstract}

Key Words: Turbulence, Numerical Analysis, Computational Fluid Dynamics, Helicity, System Rotation, Complex Helical Waves Decomposition, Scrambling Effect

\section{Introduction}

Rotating flows have numerous industrial applications in the fields of engineering, geophysics, astrophysics and meteorology. The turbulence properties of the rotating flows in blade passages of radial pumps and gas or steam turbines determine the efficiency of these devices. Therefore, the study of rotating turbulence is interesting from the viewpoints of both turbulence modeling and fundamental research ${ }^{(1),(2)}$. To date, a number of researchers have studied rotating turbulence that includes decaying homogeneous turbulence ${ }^{(3)-(6)}$, homogeneous turbulent shear flow ${ }^{(7)-(9)}$, turbulent channel flow ${ }^{(10)-(14)}$ and turbulent Couette flow $^{(15)}$ with theoretical, experimental and numerical methods. Furthermore, in order to develop a model for the rotating turbulence, it is necessary to elucidate

* Received 9th March, 2001. Japanese original: Trans. Jpn. Soc. Mech. Eng., Vol.66, No.644, B (2000), pp. 962-969 (Received 16th August, 1999)

** Department of Mechanical Engineering, Nagoya Institute of Technology, Gokiso-cho, Showa-ku, Nagoya, Aichi 466-8555, Japan. E-mail: morinisi @ cfd.mech.nitech.ac.jp the turbulence structure of the turbulent field. Helicity, an important parameter for evaluating the turbulence structure, and the relationship between the helicity and the vortex structure are investigated in this study.

With the development of computers, numerical simulation has become one of the main tools for turbulence research. In particular, direct numerical simulation (DNS) plays a significant role in the investigation of turbulence structure and turbulence modeling. When DNS of homogeneous turbulence is considered, one needs an initial turbulent velocity field. Conventionally, a fully symmetric velocity field in which the turbulence statistics satisfy the rotation and reflection symmetries ${ }^{(16)}$ is generated as an initial velocity field for the DNS of homogeneous decaying turbulence $e^{(17),(18)}$. Helicity is evidently zero if the turbulence statistics possess the reflectional symmetry property. Consequently, a fully symmetric turbulent flow field does not include mean helicity. However, when the turbulence is subjected to uniform system rotation, the reflection symmetry of the statistics is destroyed by the rotation ${ }^{(19)}$ and the flow field becomes an axisymmetric turbulent field. Hence, the turbu- 
lence subjected to uniform system rotation allows the coexistence of the shell symmetric kinetic energy and helicity spectrum. Lesieur ${ }^{(20)}$ reported that isotropy possesses rotation symmetry only, and it is referred to as semi-isotropy in some studies ${ }^{(5)}$. Hence, the definition of semi-isotropy seems to fit more suitably for actual rotating turbulence than that of isotropy. Using the turbulence closure theory of eddy-damped quasi-normal Markovian (EDQNM), Andre and Lesieur $^{(21)}$ investigated the effects of the helicity on the turbulence, and indicated that the helicity inhibits the kinetic energy transfer of the homogeneous turbulence in the stationary system. Kraichnan ${ }^{(22)}$ also predicted the same effect by theoretical analysis. Based on the definition of the helicity spectrum, i.e., $\left\langle\hat{\boldsymbol{u}} \cdot \widehat{\boldsymbol{\omega}}^{*}\right\rangle / 2$, Polifke and Shtilman ${ }^{(23)}$ proposed a random phase method to introduce the helicity into a turbulent field. Murakami et al. ${ }^{(24)}$ also used this method to study the effect of helicity on turbulence. However, this method needs much realization to obtain an effective field with less helicity and no detailed discussion on the effects of the helicity was included in their studies.

In the present study, a simple and convenient method is proposed to introduce the helicity into an initial isotropic turbulent velocity field. DNS of rotating homogeneous decaying turbulence is carried out with and without the initial helicity. Then, the effects of the system rotation and the helicity on the turbulence are investigated by theoretical analysis and based on DNS results.

\section{Nomenclature}

Roman symbols

$E(k)$ : energy spectrum function

$e(\boldsymbol{k})$ : energy spectrum

$\boldsymbol{e}^{1}, \boldsymbol{e}^{2}$ : basis vectors of Craya-Herring frame

$F$ : flatness factor of velocity gradient

$H(k)$ : helicity spectrum function

$h(\boldsymbol{k})$ : helicity spectrum

He : helicity, $\langle\boldsymbol{u} \cdot \boldsymbol{\omega}\rangle / 2$

$I$ : complex unit, $\sqrt{-1}$

$K$ : turbulence kinetic energy

$\boldsymbol{k}$ : wave number vector

$k$ : magnitude of wave number $\boldsymbol{k}$

$L_{i j, k}$ : integral lengthscale

$L_{33,3}$ : longitudinal integral length scale

$L_{11,3}+L_{22,3}:$ transverse integral length scale

$L^{*}:\left(L_{11,3}+L_{22,3}\right) / L_{33,3}$

$\boldsymbol{N}( \pm \boldsymbol{k})$ : basis vectors of complex helical waves decomposition

$\boldsymbol{n}^{k}$ : unit vector along the direction $x_{k}$

$\boldsymbol{p}$ : wave-number vector $p$ : fluctuating pressure, or magnitude of wave number $\boldsymbol{p}$

$P_{i j}:$ projection operator, $\delta_{i j}-k_{i} k_{j} / k^{2}$

$\boldsymbol{q}$ : wave-number vector

$q$ : magnitude of wave number $\boldsymbol{q}$

$q_{0}$ : initial kinetic energy (at $t=0$ )

$r$ : position vector

$R e_{\lambda}$ : Taylor microscale Reynolds number

$S$ : skewness factor of velocity gradient $s, s_{k}, s_{p}, s_{q}$ : sign of helicity mode

$T(k)$ : energy transfer function

$\boldsymbol{u}$ : velocity vector

$Z(\boldsymbol{k})$ : complex deviator spectrum

Greek symbols

$\alpha$ : relative helicity coefficient

$\delta_{i j}$ : Kronecker delta tensor

$\varepsilon_{i j l}$ : alternating tensor

$\lambda$ : Taylor microscale

$\nu$ : kinetic viscosity of working fluid

$\xi_{+1}, \xi_{-1}:$ helical modes

$\Pi$ : modified pressure

$\rho$ : fluid density

$\omega$ : vorticity vector

$\omega$ : magnitude of vorticity vector $\omega$

$\boldsymbol{\Omega}$ : angular velocity vector

$\Omega$ : magnitude of angular velocity vector $\boldsymbol{\Omega}$

Other symbols

\langle\rangle : ensemble average

( ) : coefficient of Fourier transform

\section{Formulation and Generation of Initial Helicity}

\section{1 Basic equations}

The incompressible Navier-Stokes (NS) and the continuity equations in a rotating system are used as the fundamental equations of this study.

$$
\begin{aligned}
& \frac{\partial \boldsymbol{u}}{\partial t}+2 \boldsymbol{\Omega} \times \boldsymbol{u}=-\nabla \Pi-\boldsymbol{\omega} \times \boldsymbol{u}+\nu \nabla^{2} \boldsymbol{u} \\
& \nabla \cdot \boldsymbol{u}=0,
\end{aligned}
$$

where $\Pi=p / \rho-(\boldsymbol{\Omega} \times \boldsymbol{r}) \cdot(\boldsymbol{\Omega} \times \boldsymbol{r}) / 2+\boldsymbol{u} \cdot \boldsymbol{u} / 2$. By applying the Fourier transform and the continuity condition to Eq. ( 1 ), the NS equation in wave space is obtained.

$$
\begin{aligned}
& \left(\frac{\partial}{\partial t}+\nu k^{2}\right) \hat{\boldsymbol{u}}(\boldsymbol{k})+\frac{2 \boldsymbol{\Omega} \cdot \boldsymbol{k}}{k^{2}}[\boldsymbol{k} \times \widehat{\boldsymbol{u}}(\boldsymbol{k})] \\
& =-\sum_{k-p-q=0}\left(1-\frac{\boldsymbol{k}}{k^{2}} \boldsymbol{k} \cdot\right)[\hat{\boldsymbol{\omega}}(\boldsymbol{p}) \times \widehat{\boldsymbol{u}}(\boldsymbol{q})]
\end{aligned}
$$

Here, $\boldsymbol{k}, \boldsymbol{p}$ and $\boldsymbol{q}$ are the wave-number vectors and $k^{2}=\boldsymbol{k} \cdot \boldsymbol{k}$. The second term on the left-hand side of this equation is the Coriolis term, and it is a linear term. In order to analyze the linear effect of the Coriolis term more clearly, we adopt the complex helical waves decomposition ${ }^{(25),(26)}$ to reform the NS equation in wave space. The solenoidal condition implies that the complex vector $\widehat{\boldsymbol{u}}(\boldsymbol{k})$ is located on the plane normal to the wave vector $\boldsymbol{k}$, and the following 
decomposition is useful :

$$
\begin{aligned}
& \widehat{\boldsymbol{u}}(\boldsymbol{k})=\xi_{+1}(\boldsymbol{k}) \boldsymbol{N}(\boldsymbol{k})+\xi_{-1}(\boldsymbol{k}) \boldsymbol{N}(-\boldsymbol{k}) \\
& \quad=\sum_{s= \pm 1} \xi_{s}(\boldsymbol{k}) \boldsymbol{N}(s \boldsymbol{k}) .
\end{aligned}
$$

The components $\xi_{+1}$ and $\xi_{-1}$ are respectively the plus and minus helical modes of the complex helical- wave decomposition. The orthogonal basis $\boldsymbol{N}(s \boldsymbol{k})$ is defined as

$$
\boldsymbol{N}(s \boldsymbol{k})=\boldsymbol{e}^{2}(\boldsymbol{k})-I s \boldsymbol{e}^{1}(\boldsymbol{k}) .
$$

Here,

$$
\boldsymbol{e}^{1}=\boldsymbol{k} \times \boldsymbol{a} /|\boldsymbol{k} \times \boldsymbol{a}|, \boldsymbol{e}^{2}=\boldsymbol{k} \times \boldsymbol{e}^{1} /|\boldsymbol{k}|
$$

and the wave vector $\boldsymbol{k}$ form a direct orthonormal frame called Craya-Herring frame ${ }^{(20)}$, where $\boldsymbol{a}$ is an arbitrary constant vector. Substituting the complex helical-wave decomposition term into Eq. (3), the evolution equation of the helical modes $\xi_{s_{k}}\left(s_{k}= \pm 1\right)$ is

$$
\begin{aligned}
& \left(\frac{\partial}{\partial t}+\nu k^{2}\right) \xi_{s_{k}}=\sum_{k+p+q=0} \sum_{s_{p}, s_{q}}\left(s_{p} p-s_{q} q\right) g^{*} \xi_{s_{p}}^{*} \xi_{s_{q}}^{*} \\
& \quad+I s_{k} g_{k} \xi_{s_{k} .}
\end{aligned}
$$

The right-hand side has been written in symmetric form in terms of $\boldsymbol{p}$ and $\boldsymbol{q}$. The triplet $\left(s_{k}, s_{p}, s_{q}\right)$ is $( \pm 1, \pm 1, \pm 1)$ and $g^{*}=-\left(\boldsymbol{N}_{s_{p}}^{*} \times \boldsymbol{N}_{s_{q}}^{*} \cdot \boldsymbol{N}_{s_{k}}^{*}\right) / 4$ is a geometrical factor dependent on the shape and the orientation of the wave vector $\operatorname{triad}(\boldsymbol{k}, \boldsymbol{p}, \boldsymbol{q})$ which satisfies the condition $\boldsymbol{k}+\boldsymbol{p}+\boldsymbol{q}=0$. Here, $g_{k}=2 \Omega \cos \theta_{k}$ and $\theta_{k}$ is the angle between $\boldsymbol{k}$ and $\boldsymbol{\Omega}$. In the stationary frame, since $g_{k}=0$, Eq. $(7)$ is written in the following form :

$$
\left(\frac{\partial}{\partial t}+\nu k^{2}\right) \xi_{s_{k}}=\sum_{k+p+q=0} \sum_{s_{p}, s_{q}}\left(s_{p} p-s_{q} q\right) g^{*} \xi_{s_{p}}^{*} \xi_{s_{q}}^{*} .
$$

The corresponding energy evolution equations (Eqs. ( 7 ) and ( 8 )) have the same form as

$$
\begin{aligned}
& \left(\frac{\partial}{\partial t}+2 \nu k^{2}\right)\left\langle\xi_{s_{k}} \xi_{s_{k}}^{*}\right\rangle \\
& \quad=\left\langle\sum_{k+p+q=0} \sum_{s_{k}, s_{p}, s_{q}} 2\left(s_{p} p-s_{q} q\right) \operatorname{Re}\left[g^{*} \xi_{s_{k}}^{*} \xi_{s_{p}}^{*} \xi_{s_{q}}^{*}\right]\right\rangle .
\end{aligned}
$$

Equation (9) shows that, even in the rotating frame, there is no explicit rotation term in the kinetic energy evolution equation.

\subsection{Spectral tensor}

A general form of the spectral tensor ${ }^{(5)}$ can be expressed as

$$
\begin{aligned}
& \widehat{U}_{i j}(\boldsymbol{k})=\left\langle\widehat{u}_{i}^{*}(\boldsymbol{k}) \widehat{u}_{j}(\boldsymbol{k})\right\rangle=e(\boldsymbol{k}) P_{i j}(\boldsymbol{k}) \\
& \quad+\operatorname{Re}\left[Z(\boldsymbol{k}) N_{i}(\boldsymbol{k}) N_{j}(\boldsymbol{k})\right]+I \varepsilon_{i j l} k_{l} h(\boldsymbol{k}) / k^{2},
\end{aligned}
$$

where $e(\boldsymbol{k}), h(\boldsymbol{k})$ and $Z(\boldsymbol{k})$ are respectively the spectra of energy, helicity and complex deviator, defined as

$$
\begin{aligned}
& e(\boldsymbol{k})=\frac{1}{2}\left\langle\widehat{\boldsymbol{u}}(\boldsymbol{k}) \cdot \widehat{\boldsymbol{u}}^{*}(\boldsymbol{k})\right\rangle \\
& \quad=\left\langle\xi_{+1}(\boldsymbol{k}) \xi_{+1}^{*}(\boldsymbol{k})+\xi_{-1}(\boldsymbol{k}) \xi_{-1}^{*}(\boldsymbol{k})\right\rangle, \\
& h(\boldsymbol{k})=\frac{1}{2}\left\langle\widehat{\boldsymbol{u}}(\boldsymbol{k}) \cdot \widehat{\boldsymbol{\omega}}^{*}(\boldsymbol{k})\right\rangle \\
& \quad=\left\langle k\left[\xi_{+1}(\boldsymbol{k}) \xi_{+1}^{*}(\boldsymbol{k})-\xi_{-1}(\boldsymbol{k}) \xi_{-1}^{*}(\boldsymbol{k})\right]\right\rangle, \\
& Z(\boldsymbol{k})=2\left\langle\xi_{+1}(\boldsymbol{k}) \xi_{-1}^{*}(\boldsymbol{k})\right\rangle .
\end{aligned}
$$

The complex deviator denotes a part of the anisotropy on the plane perpendicular to $\boldsymbol{k}^{(5),(24)}$. For isotropic turbulence $[Z(\boldsymbol{k})=0]$ with rotation symmetry only, the spectral tensor can be written as

$$
\hat{U}_{i j}(\boldsymbol{k})=e(k) P_{i j}(\boldsymbol{k})+I \varepsilon_{i j l} k_{l} h(k) / k^{2}
$$

where $e(\boldsymbol{k})$ and $h(\boldsymbol{k})$ are replaced by the corresponding isotropic forms $e(k)=E(k) /\left(4 \pi k^{2}\right)$ and $h(k)=$ $H(k) /\left(4 \pi k^{2}\right)$, respectively. If the reflectional symmetry is also satisfied, the spectral tensor for isotropic turbulence is simplified to:

$$
\hat{U}_{i j}(\boldsymbol{k})=e(k) P_{i j}(\boldsymbol{k}) \text {. }
$$

However, since the reflectional symmetry is destroyed by the system rotation, the general spectral tensor for the isotropic rotating turbulence has the form of Eq. (12).

Using the helical modes $\xi_{+1}$ and $\xi_{-1}$, one obtains the shell symmetry forms of the spectrum as

$$
\begin{aligned}
& e^{++}(k)=\left\langle\xi_{+1}(\boldsymbol{k}) \xi_{+1}^{*}(\boldsymbol{k})\right\rangle=\frac{E^{++}(k)}{4 \pi k^{2}}, \\
& e^{--}(k)=\left\langle\xi_{-1}(\boldsymbol{k}) \xi_{-1}^{*}(\boldsymbol{k})\right\rangle=\frac{E^{--}(k)}{4 \pi k^{2}},
\end{aligned}
$$

where the definitions of $E^{++}(k)$ and $E^{--}(k)$ are

$$
\begin{aligned}
& E^{++}(k)=\frac{1}{2}\left[E(k)+\frac{H(k)}{k}\right], \\
& E^{--}(k)=\frac{1}{2}\left[E(k)-\frac{H(k)}{k}\right] .
\end{aligned}
$$

\section{3 Generation of initial helicity}

In the present study, we propose a simple and useful method to obtain a velocity field with an arbitrary distribution of the helicity spectrum. The advantage of this method is that only the amplitudes of the helical modes are changed with a scalar parameter, the kinetic energy of the field is kept constant.

Based on Eq. (14), it can be assumed that,

$$
\begin{aligned}
& \xi_{+1}(\boldsymbol{k}, t)=\left[\frac{E^{++}(k, t)}{4 \pi k^{2}}\right]^{1 / 2} \exp \left(I \theta_{1}\right), \\
& \xi_{-1}(\boldsymbol{k}, t)=\left[\frac{E^{--}(k, t)}{4 \pi k^{2}}\right]^{1 / 2} \exp \left(I \theta_{2}\right),
\end{aligned}
$$

where $\theta_{1}$ and $\theta_{2}$ are the phase angles of $\xi_{+1}$ and $\xi_{-1}$ respectively, and vary between 0 to $2 \pi$. Here, we introduce a relative helicity coefficient

$$
\alpha(\boldsymbol{k})=\frac{h(\boldsymbol{k})}{k e(\boldsymbol{k})}=\frac{\left\langle\xi_{+1}(\boldsymbol{k}) \xi_{+1}^{*}(\boldsymbol{k})-\xi_{-1}(\boldsymbol{k}) \xi_{-1}^{*}(\boldsymbol{k})\right\rangle}{\left\langle\xi_{+1}(\boldsymbol{k}) \xi_{+1}^{*}(\boldsymbol{k})+\xi_{-1}(\boldsymbol{k}) \xi_{-1}^{*}(\boldsymbol{k})\right\rangle} .
$$

Here, $\alpha=+1$ and -1 correspond to the presence of the plus and minus modes only and the helicity takes the maximum and minimum values, respectively. $\alpha=$ 0 corresponds to a non-helicity field. Equations (15) and (17) yield, at a certain time $t_{0}$,

$$
H\left(k, t_{0}\right)=\alpha k E\left(k, t_{0}\right),|\alpha| \leq 1 .
$$

In accordance with Eq. (18), the helical modes are brought into : 


$$
\begin{aligned}
& \xi_{+1}^{\alpha}\left(\boldsymbol{k}, t_{0}\right)=\left[\frac{(1+\alpha) E\left(k, t_{0}\right)}{8 \pi k^{2}}\right]^{1 / 2} \exp \left(I \theta_{1}\right), \\
& \xi_{-1}^{\alpha}\left(\boldsymbol{k}, t_{0}\right)=\left[\frac{(1-\alpha) E\left(k, t_{0}\right)}{8 \pi k^{2}}\right]^{1 / 2} \exp \left(I \theta_{2}\right) .
\end{aligned}
$$

A direct deduction of this equation yields :

$$
\begin{aligned}
& \xi_{+1}^{\alpha}\left(\boldsymbol{k}, t_{0}\right)=(1+\alpha)^{\frac{1}{2}} \xi_{+1}^{0}\left(\boldsymbol{k}, t_{0}\right), \\
& \xi_{-1}^{\alpha}\left(\boldsymbol{k}, t_{0}\right)=(1-\alpha)^{\frac{1}{2}} \xi_{-1}^{0}\left(\boldsymbol{k}, t_{0}\right)
\end{aligned}
$$

The superscripts $\alpha$ and 0 denote that the relative helicity coefficients have nonzero and zero values, respectively. If an initial field at a certain time $t_{0}$ is known, i.e., the helical modes $\xi_{+1}^{0}$ and $\xi_{-1}^{0}$ are known, one finds it easy to introduce the helicity into this field through Eq. (20) by only changing the parameter $\alpha$. The corresponding kinetic energy of Eq. (20) is

$$
e^{\alpha}\left(\boldsymbol{k}, t_{0}\right)=e^{0}\left(\boldsymbol{k}, t_{0}\right)+\alpha\left\langle\left|\xi_{+1}^{0}\right|^{2}-\left|\xi_{-1}^{0}\right|^{2}\right\rangle .
$$

This means that, when the helicity is generated, a part of the extra energy $\alpha\left\langle\left|\xi_{+1}^{0}\right|^{2}-\left|\xi_{-1}^{0}\right|^{2}\right\rangle$ is also introduced into the initial field. The kinetic energy is conserved only when the field satisfies the condition $\left|\xi_{+1}^{0}\right|=\left|\xi_{-1}^{0}\right|$. However, in the numerical simulation in particular, it is difficult to satisfy the condition exactly when we use a finite number of Fourier modes. In order to conserve the kinetic energy of the system, we reform Eq.

(20) into the following forms:

$$
\left.\begin{array}{l}
\xi_{+1}^{\alpha}\left(\boldsymbol{k}, t_{0}\right)=(1+\alpha \beta)^{1 / 2} \xi_{+1}^{0}\left(\boldsymbol{k}, t_{0}\right), \\
\xi_{-1}^{\alpha}\left(\boldsymbol{k}, t_{0}\right)=(1-\alpha)^{1 / 2} \xi_{-1}^{0}\left(\boldsymbol{k}, t_{0}\right), \\
\xi_{+1}^{\alpha}\left(\boldsymbol{k}, t_{0}\right)=(1+\alpha)^{1 / 2} \xi_{+1}^{0}\left(\boldsymbol{k}, t_{0}\right), \\
\xi_{-1}^{\alpha}\left(\boldsymbol{k}, t_{0}\right)=(1-\alpha / \beta)^{1 / 2} \xi_{-1}^{0}\left(\boldsymbol{k}, t_{0}\right),
\end{array}\right\}(-1 \leq \alpha \leq 0)
$$

where $\beta=\left(\xi_{-1}^{0}\right)^{2} /\left(\xi_{+1}^{0}\right)^{2}$. In this paper, we use Eq. (22) to introduce the helicity into a given isotropic turbulent field. Then, the velocity field in Fourier space is computed by using the definition of the complex helical-wave decomposition given by Eq. ( 4 ).

\section{Initial Condition}

In this study, we perform DNS of the decaying turbulence subjected to system rotation by using a new DNS algorithm ${ }^{(6)}$, which integrates the Coriolis term analytically. The initial flow field for the DNS is obtained by a pre-computation. The initial flow field of the precomputations is produced through Eq. (19) with $t_{0}=0, \alpha=0$, and $\theta_{1}$ and $\theta_{2}$ are random numbers varying between 0 to $2 \pi$. The initial energy spectrum is $^{(27)}$

$$
E(k, 0)=\frac{32}{3} \sqrt{\frac{2}{\pi}} q_{0} \frac{k^{4}}{k_{p}^{5}} \exp \left(-2 \frac{k^{2}}{k_{p}^{2}}\right),
$$

with $q_{0}=13.5$ and $k_{p}=8$. Then, the precomputation is performed to $t_{0}=2.5[\mathrm{~s}]$. At $t_{0}=2.5[\mathrm{~s}]$, reliable velocity gradient skewness and power-law decay for the turbulent kinetic energy are obtained. Finally, the turbulent velocity field at $t_{0}$ is adopted as the initial flow field without the helicity and the initial flow field with helicity is generated by using Eq. (22) and $\alpha \neq 0$.
Table 1 Statistics of initial turbulent field

\begin{tabular}{|c|c|c|c|c|c|c|}
\hline$\alpha$ & $K_{0}$ & $\varepsilon_{0}$ & $H e_{0}$ & $\operatorname{Re}_{\lambda}$ & $S$ & $F$ \\
\hline 0 & 0.2013 & 0.0567 & 0.0067 & 53.4 & -0.496 & 4.680 \\
\hline 1 & 0.2013 & 0.0567 & 1.6990 & 53.4 & -0.080 & 4.258 \\
\hline-1 & 0.2013 & 0.0567 & -1.6990 & 53.4 & -0.061 & 4.310 \\
\hline
\end{tabular}

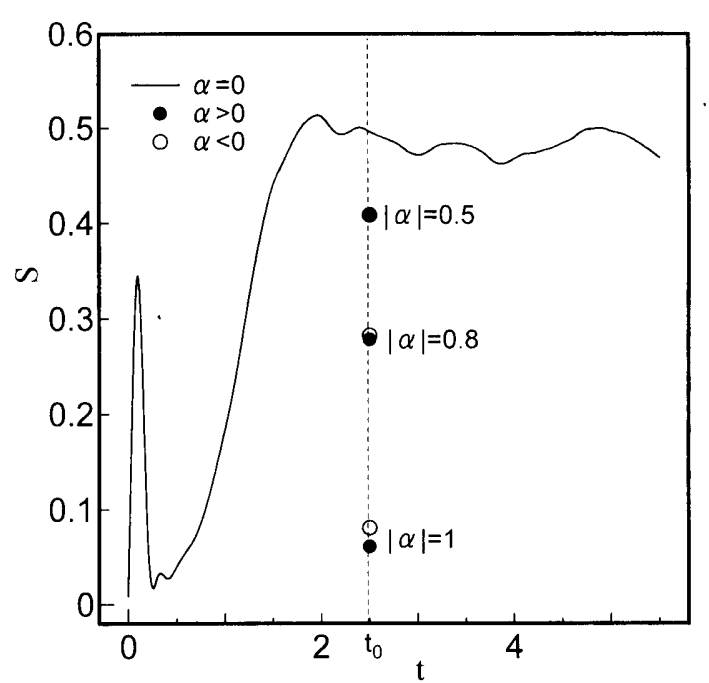

Fig. 1 Evolution of velocity gradient skewness factor

The computational region is $(2 \pi \times 2 \pi \times 2 \pi)$ and periodic conditions are assumed in all directions. The spectral mode number is 128 in each direction. The kinetic viscosity and the time increment are set as $\nu=0.001\left[\mathrm{~m}^{2} / \mathrm{s}\right]$ and $\Delta t=0.01[\mathrm{~s}]$, respectively. DNS of decaying homogenous turbulence for $\alpha=0$ and 1 is carried out with $\Omega=0,0.5,1,2$ and $5[\mathrm{rad} / \mathrm{s}]$, respectively. In order to investigate the influence of the minimum helicity, DNS of $\alpha=-1$ with $\Omega=0$ and 2 is also submitted. The statistics of the initial field at $t_{0}=2.5[\mathrm{~s}]$ are presented in Table 1. Here, $K_{0}\left[\mathrm{~m}^{2} / \mathrm{s}^{2}\right]$, $\varepsilon_{0}\left[\mathrm{~m}^{2} / \mathrm{s}^{3}\right]$ and $H e_{0}\left[\mathrm{~m} / \mathrm{s}^{2}\right]$ are the initial kinetic energy, energy dissipation rate and helicity, respectively.

Table 1 shows that there is slight initial helicity in the initial turbulent field for the case of $\alpha=0$. This comes from the turbulent field obtained by the precomputation. The corresponding relative helicity coefficient of this turbulent field is $\alpha_{0}=0.00268$. In the present study, this small $\alpha_{0}$ value can be ignored and considered as $\alpha=0$. Additionally, Fig. 1 also shows that the absolute value of the velocity gradient skewness factor decreases with increasing the value of $\alpha$. This can be explained by the definition of the skewness factor. The definition of the skewness factor for the velocity gradient is given by

$$
S=\left\langle\left(\frac{\partial u_{i}}{\partial x_{j}}\right)^{3}\right\rangle /\left\langle\left(\frac{\partial u_{i}}{\partial x_{j}}\right)^{2}\right\rangle^{3 / 2} .
$$

Batchelor and Townsend ${ }^{(28)}$ have shown that, for isotropic three-dimensional turbulence, the skewness factor $S$ is equivalent to 


$$
S=\left(\frac{135}{98}\right)^{1 / 2}\left\langle\omega_{i} \omega_{j} \frac{\partial u_{i}}{\partial x_{j}}\right\rangle / D^{3 / 2},
$$

with $D=\left\langle\omega^{2}\right\rangle / 2$. Using the homogeneous condition, $\left\langle\partial() / \partial x_{i}\right\rangle=0$, Eq. (25) can be brought into a vector form :

$$
S=\left(\frac{135}{98}\right)^{1 / 2}\langle\boldsymbol{\omega} \cdot \nabla \times(\boldsymbol{u} \times \boldsymbol{\omega})\rangle / D^{3 / 2} .
$$

Helicity is defined as $\langle\boldsymbol{u} \cdot \boldsymbol{\omega}\rangle / 2$, and it takes the maximum absolute value when the velocity vector $\boldsymbol{u}$ is parallel or antiparallel to the vorticity vector $\omega$. On the other hand, while $\boldsymbol{u}$ is perpendicular to $\boldsymbol{\omega}$, the helicity and the term $\boldsymbol{u} \times \boldsymbol{\omega}$ take the minimum and the maximum absolute values, respectively. One can find from Eq. (26) that, when the helicity is increased, $\boldsymbol{u}$ becomes parallel or antiparallel to $\boldsymbol{\omega}$ and the value of $|\boldsymbol{u} \times \boldsymbol{\omega}|$ decreases. Hence, the absolute value of the skewness factor decreases with increasing the helicity.

\section{Effects of System Rotation and Helicity}

\section{1 Effects of system rotation}

Figure 2 shows the evolution of the turbulence kinetic energy with system rotation. It shows that the kinetic energy decays with time and the decay rate is decreased by the system rotation. The effects of the system rotation on the turbulence are discussed analytically as follows.

The system for the helical modes corresponding to the single triplet $\left(s_{k}, s_{p}, s_{q}\right)$ have the form (from Eq.

( 7 ) with viscosity omitted),

$$
\begin{aligned}
& \dot{\xi}_{s_{k}}=\left(s_{p} p-s_{q} q\right) g^{*} \xi_{s_{p}}^{*} \xi_{s_{q}}^{*}+I s_{k} g_{k} \xi_{s_{k}}, \\
& \dot{\xi}_{s_{p}}=\left(s_{q} q-s_{k} k\right) g^{*} \xi_{s_{q}}^{*} \xi_{s_{k}}^{*}+I s_{p} g_{p} \xi_{s_{p}}, \\
& \dot{\xi}_{s_{q}}=\left(s_{k} k-s_{p} p\right) g^{*} \xi_{s_{k}}^{*} \xi_{s_{p}}^{*}+I s_{q} g_{q} \xi_{s_{q} .} .
\end{aligned}
$$

Here, a dot denotes the time derivative. The first term on the right-hand side (RHS) is the nonlinear term and the second term is a linear term introduced

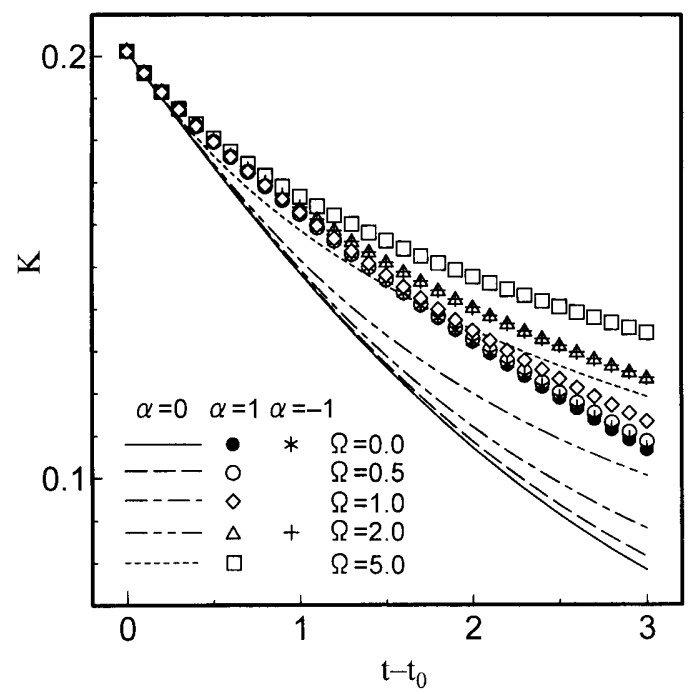

Fig. 2 Evolution of turbulence kinetic energy by the system rotation. The system without rotation introduced by Waleffe ${ }^{(26)}$ can be recovered from Eq. (27) with $g_{k}=g_{p}=g_{q}=0$. In general, following Eq. (27), the turbulence kinetic energy is cascaded from the low to high wave numbers due to the nonlinear interactions among wave vectors $(\boldsymbol{k}, \boldsymbol{p}, \boldsymbol{q})$. However, one can find from the following analysis that system rotation affects the nonlinear interactions significantly, although this effect is implicit. When the rotation is strong enough, the effect of the nonlinear interactions can be ignored, compared with the influence of the Coriolis term. According to the linear system of Eq. (27), the linear solution corresponding to the helical mode $\xi_{s_{k}}$ is

$$
\xi_{s_{\boldsymbol{k}}}(\boldsymbol{k}, t)=\xi_{s_{\boldsymbol{k}}}(\boldsymbol{k}, 0) \exp \left(I s_{k} g_{k} t\right)
$$

In order to investigate the nonlinear interactions in the rotating case, we introduce the following assumption $^{(5)}$ :

$$
\xi_{s_{k}}(\boldsymbol{k}, t)=a_{s_{k}}(\boldsymbol{k}, t) \exp \left(I s_{k} g_{k} t\right),
$$

where $a_{s_{k}}(\boldsymbol{k}, t)$ expresses the nonlinear contribution. One can recover the linear solution of Eq. (28) with $a_{s_{k}}(\boldsymbol{k}, t)=\xi_{s_{\boldsymbol{k}}}(\boldsymbol{k}, 0)$. Then, substitution of Eq. (29) into Eq. (27) yields the equation for the nonlinear component $a_{s_{k}}(\boldsymbol{k}, t)$ :

$$
\begin{aligned}
& \dot{a}_{s_{k}}(\boldsymbol{k}, t)=\left(s_{p} p-s_{q} q\right) g^{*} a_{s_{p}}^{*} a_{s_{q}}^{*} \\
& \quad \times \exp \left[-I\left(s_{k} g_{k}+s_{p} g_{p}+s_{q} g_{q}\right) t\right]:
\end{aligned}
$$

This means that if $s_{k} g_{k}+s_{p} g_{p}+s_{q} g_{q} \neq 0$, then the term $\exp \left[-I\left(s_{k} g_{k}+s_{p} g_{p}+s_{q} g_{q}\right) t\right]$ provides a strong rotation to the nonlinear interactions with the time evolution and the nonlinear effect is averaged out over a long time-scale. This rotation effect is called the 'scrambling effect' in the literature ${ }^{(4)}$. Hence, in addition to the limit $\boldsymbol{k}+\boldsymbol{p}+\boldsymbol{q}=0$ on the triad interaction (nonlinear interaction), the following limit also acts on

$$
s_{k} g_{k}+s_{p} g_{p}+s_{q} g_{q}=0,
$$

or equivalently,

$$
2 \Omega \cdot\left(s_{k} \boldsymbol{k} / k+s_{p} \boldsymbol{p} / p+s_{q} \boldsymbol{q} / q\right)=0 .
$$

The triad interaction that satisfies Eq. (32) is called the 'resonant triad interaction'(6). A direct deduction from the above analysis is that only the resonant triad interactions contribute to the energy transfer in a rotating system while all the triad interactions in a nonrotating system contribute to the energy transfer. Hence, the transfer rate in a nonrotating system is generally larger than that in the rotating system. Figure 3 shows the kinetic energy transfer function $T(k, t)$ with $\alpha=0 . T(k, t)$ is defined as

$$
\begin{aligned}
& T(k, t)=\int\left[-P_{i j m}(\boldsymbol{k})\right. \\
& \quad \times \int_{\boldsymbol{k}-\boldsymbol{p}-\boldsymbol{q}=\mathbf{0}} \operatorname{Im}\left\langle\widehat{u}_{i}(\boldsymbol{k}, t) \widehat{u}_{j}(\boldsymbol{p}, t)\right. \\
& \left.\left.\quad \times \widehat{u}_{m}(\boldsymbol{q}, t)\right\rangle d \boldsymbol{p} d \boldsymbol{q}\right] d A(\boldsymbol{k}),
\end{aligned}
$$

where $P_{i j m}(\boldsymbol{k})=k_{m} P_{i j}(\boldsymbol{k})+k_{j} P_{i m}(\boldsymbol{k})$, and $\int d A(\boldsymbol{k})$ 


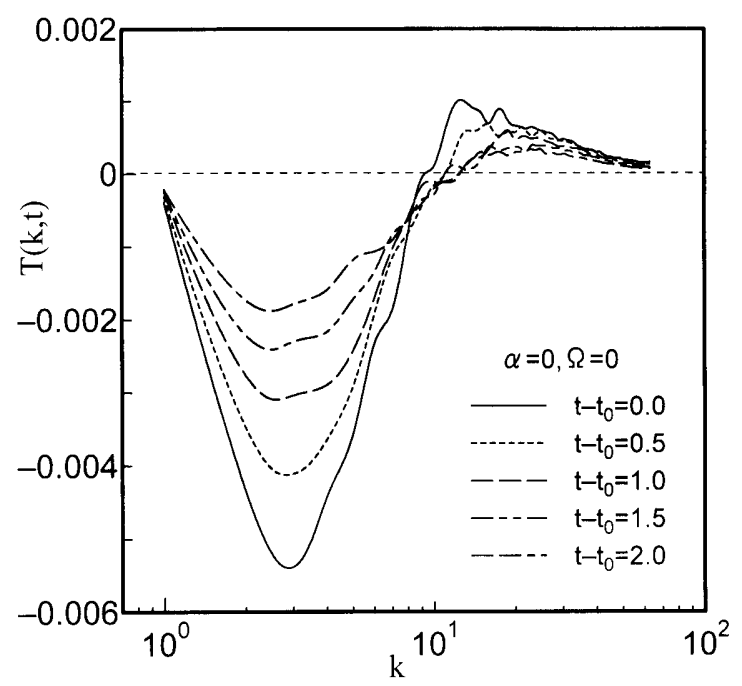

Fig. 3 Evolution of energy transfer function

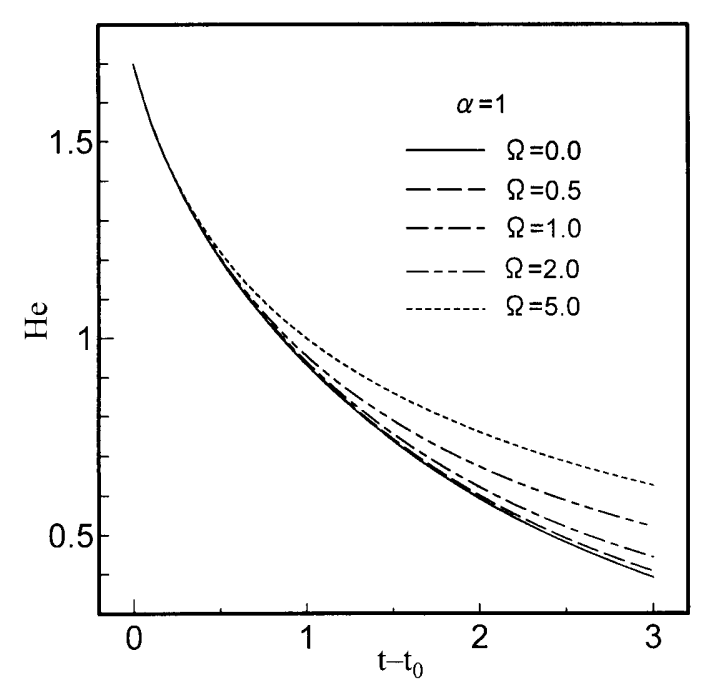

Fig. 5 Evolution of helicity

denotes the integration about the shell of radium $k$. Figure 4 shows that the absolute value of $T(k, t)$ decreases with the increase of the rotation rates. This DNS result is also consistent with the result of previous analysis. Since the absolute value is decreased by rotation, the energy cascade from the low to high wave numbers is inhibited.

In addition, since Eq. (29) is an exact expression of the helical mode $\xi_{s_{k}}$ which includes the nonlinear effect, one finds that not only the kinetic energy, but also the helicity and the complex deviator can be determined by this equation. With the same analysis of energy transfer, one can deduce that there is scrambling effect on the evolutions of $h$ and $|Z|$, respectively. In other words, the decay of the helicity and the complex deviator are also inhibited by system rotation. As an example, the evolution of the helicity $\mathrm{He}$ is presented in Fig. 5. It shows that the decay rate of the helicity decreases with the increase of the rotation

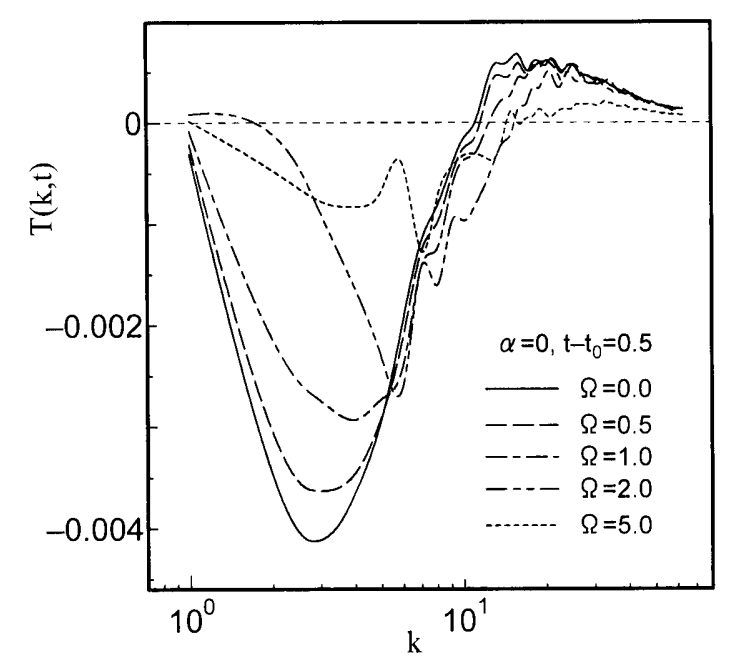

Fig. 4 Effect of rotation on energy transfer function

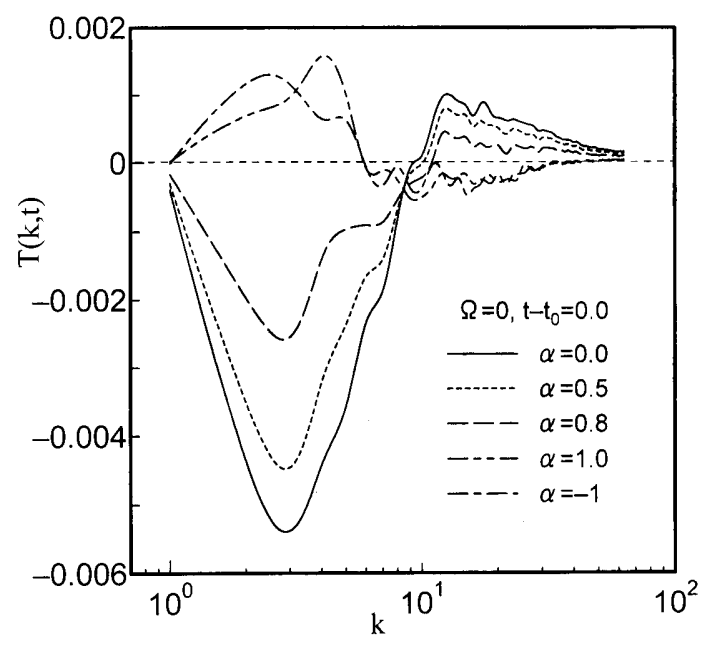

Fig. 6 Effect of helicity on energy transfer function

rate.

\section{2 Effect of helicity}

As shown in Fig. 2, even with the same rotation rate, the energy decay of the cases with helicity is slower than that of the cases without the mean helicity. That is, the presence of helicity also inhibits the energy decay. Figure 6 shows the effect of the helicity on $T(k, t)$. With the increase of the absolute value of $\alpha$, the value of $T(k, t)$ decreases gradually. In particular, the reverse cascade is presented in the cases of $|\alpha|=1$. In accordance with the definition of $T(k, t)$, the effect of the helicity on $T(k, t)$ can be explained as follows. Equation (33) can be rewritten in a more symmetric form,

$$
T(k)=2 \int \operatorname{Re}\left\langle\hat{\boldsymbol{\lambda}}(\boldsymbol{k}) \cdot \hat{\boldsymbol{u}}^{*}(\boldsymbol{k})\right\rangle d A(\boldsymbol{k}) .
$$

Here, $\lambda=\boldsymbol{u} \times \boldsymbol{\omega}$ is the Lamb vector., From this equation, considering the definition of the helicity, with the increase of the helicity, velocity $\boldsymbol{u}$ and vorticity $\boldsymbol{\omega}$ 


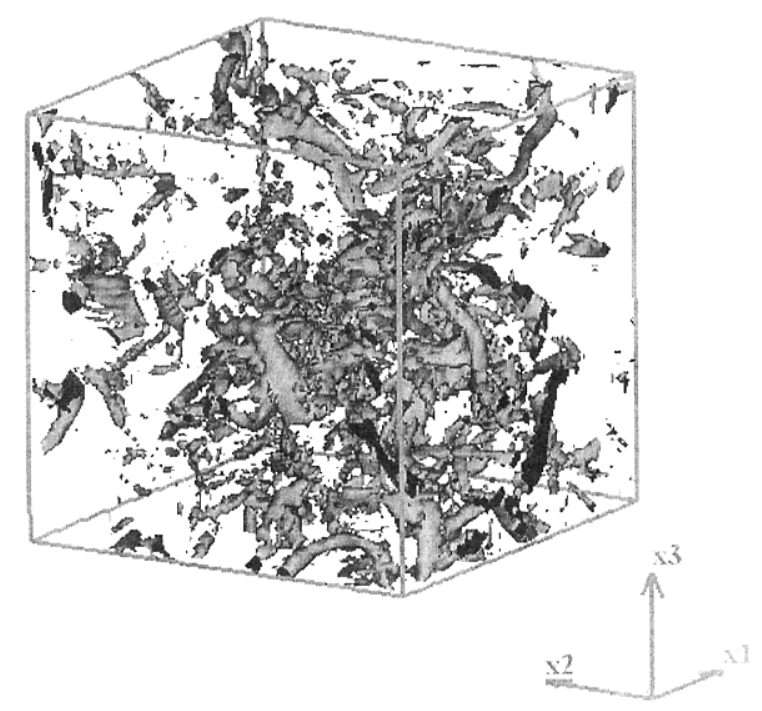

(a) $\quad \alpha=0, t-t_{0}=0[\mathrm{~s}]$

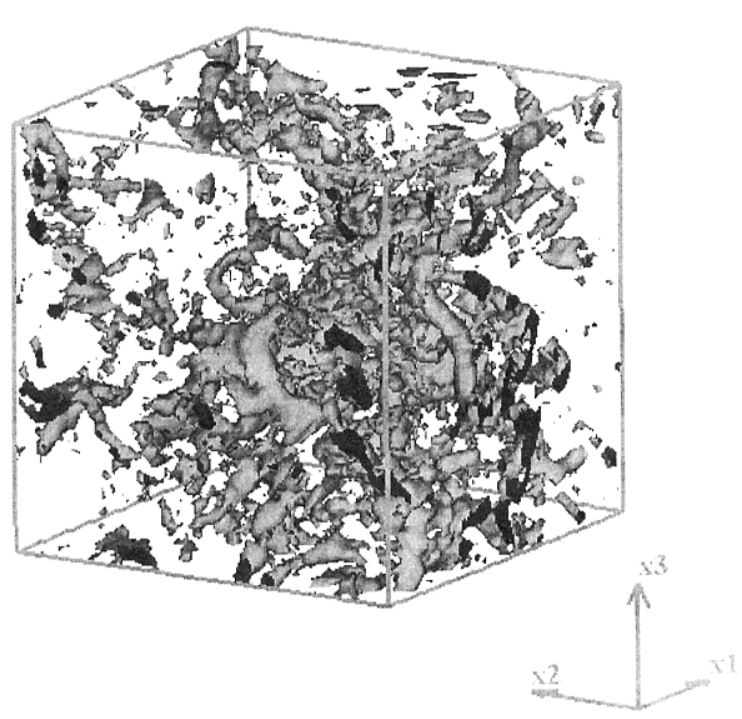

(b) $\quad \alpha=1, t-t_{0}=0[\mathrm{~s}]$

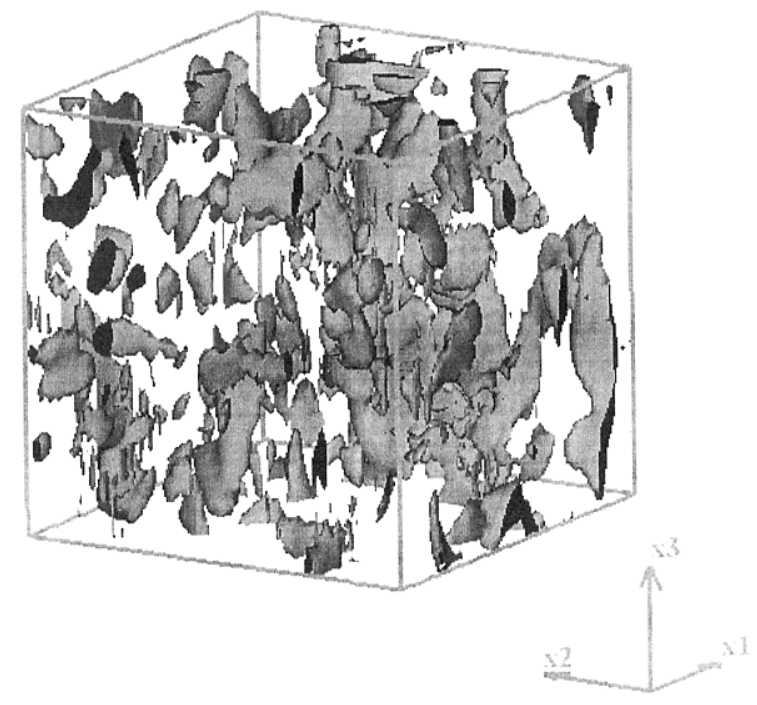

(c) $\quad \alpha=0, t-t_{0}=6[\mathrm{~s}]$

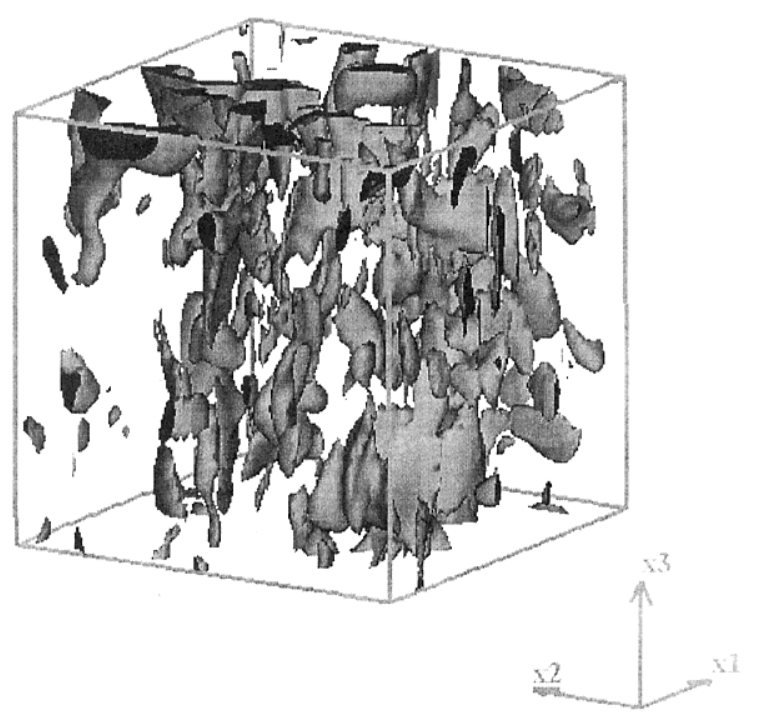

(d) $\quad \alpha=1, t-t_{0}=6[\mathrm{~s}]$

Fig. 7 Isosurface of vorticity $(\Omega=5[\mathrm{rad} / \mathrm{s}])$

travel parallel to each other, which leads us to deduce that the value of $\lambda$ decreases. Hence, the energy transfer function will decrease with the iilcrease of the helicity. It is consistent with the DNS result shown in Fig. 5.

\subsection{Turbulence structure}

Figure 7 presents the isosurface of the absolute value of the turbulent vorticity. It is well known that two-dimensionalization is one of the effects of system rotation. That is, the vortical structure is stretched and elongated along the rotation axis by system rotation. As shown in Fig. 7 ( c ), such a vortical structure does appear in the rotating system without the initial helicity $(\alpha=0)$. On the other hand, the two-dimensionalization is obviously weakened by the presence of helicity [Fig. 7(d), $\alpha=1$ ]. The effect of the helicity on the vortical structure of the rotating system can be demonstrated by the integral length scale. In Fig. 8, the evolution of the ratio $L^{*}$ of the transverse integral length scale to the longitudinal one is presented. The integral length scales $L_{i j, k}$ and $L^{*}$ are defined as

$$
\begin{aligned}
& L_{i j, k}=\int_{0}^{\infty}\left\langle u_{i}(x) u_{j}\left(x+r \boldsymbol{n}^{k}\right)\right\rangle d r \mid\left\langle u_{i}(x) u_{j}(x)\right\rangle, \\
& L^{*}=\left(L_{11,3}+L_{22,3}\right) / L_{33,3} .
\end{aligned}
$$

The value of $L^{*}$ in the figure is normalized by its initial value at $t-t_{0}=0$. The ratio of the integral lengthscale in the inertial frame $(\Omega=0)$ undergoes almost no change with time, irrespective of whether or not the initial field has helicity. On the other hand, in the rotating system, $L^{*}$ with $\alpha=0$ increases significantly while $L^{*}$ with $\alpha=1$ increases more slowly. Cambon et al. ${ }^{(5)}$ defined the longitudinal and the transverse two-dimensional energy components as 


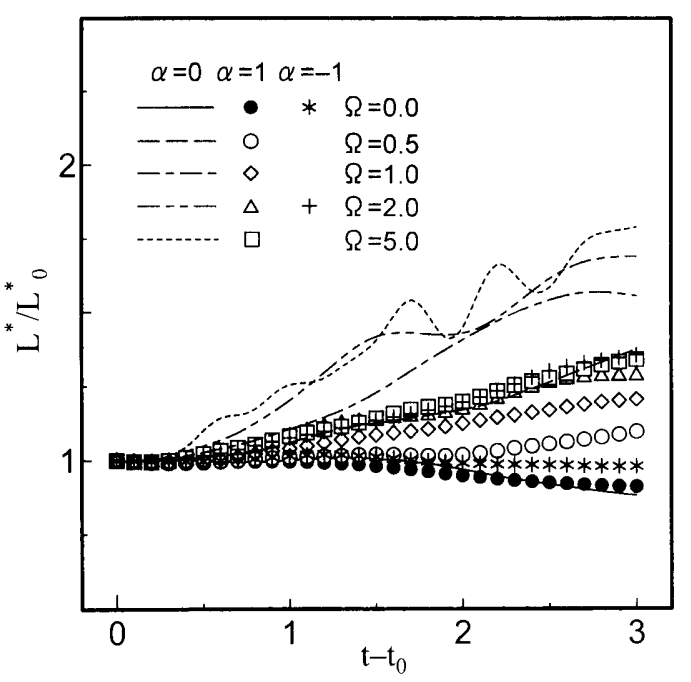

Fig. 8 Evolution of integral lengthscale

$$
\begin{aligned}
& E_{33,3}=\left\langle u_{3}^{2}\right\rangle L_{33,3}=\left.\pi \int(e+\operatorname{Re}[Z])\right|_{k_{3}=0} d \boldsymbol{k}^{2}, \\
& E_{11,3}+E_{22,3}=\left\langle u_{1}^{2}\right\rangle L_{11,3}+\left\langle u_{2}^{2}\right\rangle L_{22,3} \\
& =\left.\pi \int(e-\operatorname{Re}[Z])\right|_{k_{3}=0} d \boldsymbol{k}^{2} .
\end{aligned}
$$

Then, these are rewritten as

$$
\begin{aligned}
& L_{33,3}=\left.\pi \int(e+\operatorname{Re}[Z])\right|_{k_{3}=0} d \boldsymbol{k}^{2} /\left\langle u_{3}^{2}\right\rangle, \\
& L_{11,3}+L_{22,3}=\left.\pi \int(e-\operatorname{Re}[Z])\right|_{k_{3}=0} d \boldsymbol{k}^{2} /\left\langle u_{1}^{2}\right\rangle,
\end{aligned}
$$

with the assumption that $\left\langle u_{1}^{2}\right\rangle \cong\left\langle u_{2}^{2}\right\rangle$. From Eqs. (11) and (17), one finds that $|Z|=0$ for the cases of $|\alpha|=1$. Hence, if the system has the maximum (plus or minus) helicity, the extent of increase of the longitudinal integral length scale is almost the same as that of the transverse integral length scale, and the two-dimensionalization by the system rotation will not appear even in the rotating system. However, when $\alpha=0$, it is difficult to obtain a turbulent field with $\operatorname{Re}[Z]=0$ by the numerical method. According to Eqs. (39) and (40), the increase of the integral length scale generally depends on the distribution of $e$ and $\operatorname{Re}[Z]$.

\section{Conclusions}

In order to investigate the effects of system rotation and helicity, first, a new method that introduces the helicity into an isotropic turbulent field for DNS is proposed. Then, DNS of rotating homogeneous decaying turbulence with or without initial helicity is carried out. The main conclusions of this study are as follows :

(1) Although both system rotation and helicity inhibit energy decay, the mechanisms are different. System rotation limits the triad interactions based on the 'scrambling effect,' while helicity reduces the energy transfer function directly.

(2) The scrambling effect also acts on the evolu- tion of helicity, in the same manner as it acts on the turbulence kinetic energy.

( 3 ) In the case without helicity, the vortex structure is apparently stretched along the rotation axis, and this tendency is weakened by the presence of initial helicity. This effect can be explained by the theoretical analysis for the integral length scale.

\section{References}

(1) Hopfinger, E.J., Rotating Fluids in Geophysical and Industrial Applications, (1992), pp. 1-378, Springer-Verlag.

(2) Nakabayashi, K., Kitoh, O. and Morinishi, Y., Effects of System Rotation on Turbulence, Trans. Jpn. Soc. Mech. Eng., (in Japanese), Vol. 65, No. 631, B (1999), pp. 815-822.

(3) Jacquin, L., Leuchter, O., Cambon, C. and Mathieu, J., Homogeneous Turbulence in the Presence of Rotation, J. Fluid. Mech., Vol. 220 (1990), pp. 1 52.

(4) Mansour, N.N., Cambon, C. and Speziale, C.G., Single Point Modeling of Initially Isotropic Turbulence under Uniform Rotation, Center for Turbulence Research Annual Research Briefs 1991, Stanford Univ./NASA Ames., (1992), pp. 159-167.

( 5 ) Cambon, C., Mansour, N.N. and Godeferd, F.S., Energy Transfer in Rotating Turbulence, J. Fluid Mech., Vol. 337 (1997), pp. 303-332.

(6) Morinishi, Y., Nakabayashi, K. and Ren, S.Q., A New DNS Algorithm for Rotating Homogeneous Decaying Turbulence, Int. J. Heat and Fluid Flow, Vol. 22, Issue 1 (2001), pp. 30-38.

( 7 ) Bardina, J., Ferziger, J.H. and Reynolds, W.C., Improved Turbulence Models Based on Large Eddy Simulation of Homogeneous, Incompressible, Turbulent Flows, Tech. Rep. TF 19, Stanford University (1983), pp. 1-174.

(8) Metais, O., Flores, C., Yanase, S., Riley, J.J. and Lesieur, M., Rotating Free-Shear Flows, Part 2. Numerical Simulations, J. Fluid Mech., Vol. 293 (1995), pp. 4780.

(9) Salhi, A. and Cambon, C., An Analysis of Rotating Shear Flow using Linear Theory and DNS and LES Results, J. Fluid Mech., Vol.347 (1997), pp. 171-195.

(10) Johnston, J.P., Halleen, R.M. and Lezius, D.K., Effects of Spanwise Rotation on the Structure of Two-Dimensional Fully Developed Turbulent Channel Flow, J. Fluid Mech., Vol.56, No. 3 (1972), pp. 533-557.

(11) Nakabayashi, K., Kitoh, O. and Konishi, Y., Turbulent Characteristics of Fully Developed TwoDimensional Channel Flow with System Rotation, Trans. Jpn. Soc. Mech. Eng., (in Japanese), Vol. 60, No. 571, B (1994), pp. 849-856.

(12) Nakabayashi, K. and Kitoh, O., Low Reynolds Number Fully Developed Two-Dimensional Channel Flow with System Rotation, J. Fluid Mech., Vol. 315 (1996), pp. 1-29. 
(13) Miyake, Y. and Kajishima, T., Numerical Simulation of the Effects of Coriolis Force on the Structure of the Turbulence, Trans. Jpn. Soc. Mech. Eng., (in Japanese), Vol. 52, No.474, B (1985), pp. 759-771.

(14) Kristoffersen, R. and Anderson, H.I., Direct Simulations of Low-Reynolds Number Turbulent Flow in a Rotating Channel, J. Fluid Mech., Vol. 256 (1993), pp. 163-197.

(15) Bech, K.H., Tillmark, N. and Andersson, H.I., An Investigation of Turbulent Plane Couette Flow at Low Reynolds Numbers, J. Fluid Mech., Vol. 347 (1997), pp. 289-314.

(16) Batchelor, G.K., Theory of Homogeneous Turbulence, (1953), pp. 40-54, Cambridge University Press.

(17) Rogallo, R.S., Numerical Experiments in Homogeneous Turbulence, NASA Tech. Memo. TM81315 (1981), pp. 1-91.

(18) Mansour, N.N. and Wray, A.A., Decay of Isotropic Turbulence at Low Reynolds Number, Phys. Fluids, Vol. 6, No. 2 (1994), pp. 808-814.

(19) Kassinos, S.C. and Reynolds W.C., A StructureBased Model for the Rapid Distortion of Homogeneous Turbulence, Tech. Rep. TF-61, Stanford University, (1994), pp. 373-387.

(20) Lesieur, M., Turbulence in Fluids (Third Revised and Enlarged Edition), (1997), pp. 141-154, Kluwar Academic Publishers, The Netherlands.
(21) Andre, J.C. and Lesieur, M., Influence of Helicity on the Evolution of Isotropic Turbulence at High Reynolds Number, J. Fluid Mech., Vol. 81, No. 1 (1977), pp. 187-207.

(22) Kraichnan, R.H., Helical Turbulence and Absolute Equilibrium, J. Fluid. Mech., Vol.59, No. 4 (1973), pp. 745-752.

(23) Polifke, W. and Shtilman, L., The Dynamics of Helical Decaying Turbulence, Phys. Fluids, Vol. A1, No. 12 (1989), pp. 2025-2033.

(24) Murakami, Y., Shtilman, L. and Levich, E., Reducing Turbulence by Phase Juggling, Phys. Fluids, Vol. A4, No. 8 (1992), pp. 1776-1781.

(25) Cambon, C. and Jacquin, L., Spectral Approach to Non-Isotropic Turbulence Subjected to Rotation, J. Fluid Mech., Vol. 202 (1989), pp. 295-317.

(26) Waleffe, F., The Nature of Triad Interactions in Homogeneous Turbulence, Phys. Fluids, Vol. A4, No. 2 (1992), pp. 350-363.

(27) Squires, K.D., Chasnov, J.R., Mansour, N.N. and Cambon, C., Investigation of the Asymptotic State of Rotating Turbulence using Large-Eddy Simulation, Center for Turbulence Research Annual Research Briefs-1993, Stanford Univ. /NASA Ames., (1994), pp. 157-170.

(28) Batchelor, G.K. and Townsend, A.A., Decay of Vorticity in Isotropic Turbulence, Proc. Roy. Soc., Vol. A191 (1947), pp. 534-550. 